\title{
On the Utility of Entanglement in Quantum Neural Computing
}

\author{
Dan Ventura \\ Brigham Young University \\ Department of Computer Science \\ Provo, UT 84602 USA \\ ventura@cs.byu.edu \\ http://axon.cs.byu.edu/Dan
}

\begin{abstract}
Efforts in combining quantum and neural computation are briefly discussed and the concept of entanglement as it applies to this subject is addressed. Entanglement is perhaps the least understood aspect of quantum systems used for computation, yet it is apparently most responsible for their computational power. This paper argues for the importance of understanding and utilizing entanglement in quantum neural computation.
\end{abstract}

\section{Introduction}

There exist at least two motivations (from the computational standpoint) for applying the unique capabilities of quantum computation to the field of neural networks:

1) to compensate for ever-decreasing scales in hardware development;

2) to produce computational capability not available using classical neural computation.

Motivation (1) is the result of Moore's Law - as hardware continues to shrink, we rapidly approach the limit of classical mechanics. When this limit is reached, individual computing components will be so small that their behavior is governed by the rules of quantum rather than classical mechanics. Motivation (2) follows naturally from the fact that quantum systems have been shown to be capable of computation that is not possible on classical systems [1] [2] [3] [4]. Are there also problems in computational learning for which quantum computation will prove superior to classical approaches? While both motivations are important, it is the second that drives the arguments presented here - given the motivation to produce superclassical computational capability, understanding and utilizing the quantum mechanical characteristic of entanglement is important for combining quantum computation with neural computation. In the following sections the basics of quantum computation are reviewed; a brief overview of current research in combining quantum with neural computing is given; the concept of entanglement is defined; and the utility of entanglement in quantum neural computing is discussed.

\section{Quantum Computation}

Quantum computation is based upon physical principles from the theory of quantum mechanics (QM), which is in many ways counterintuitive. Yet it has provided us with perhaps the most accurate physical theory (in terms of predicting experimental results) ever devised by science. The theory is well established and is covered in its basic form by many textbooks (see for example [5]). Several necessary ideas that form the basis for the study of quantum computation are briefly reviewed here.

\subsection{Linear Superposition}

Linear superposition is closely related to the familiar mathematical principle of linear combination of vectors. Quantum systems are described by a wave function $\psi$ that exists in a Hilbert space. The Hilbert space has a set of states, $\left|\phi_{i}\right\rangle$, that form a basis, and the system is described by a quantum state $|\psi\rangle$,

$$
|\psi\rangle=\sum_{i} c_{i}\left|\phi_{i}\right\rangle
$$

$|\psi\rangle$ is said to be in a linear superposition of the basis states $\left|\phi_{i}\right\rangle$, and in the general case, the coefficients $c_{i}$ may be complex. Use is made here of the Dirac bracket notation, 
where the ket $|\cdot\rangle$ is analogous to a column vector, and the bra $\langle\cdot|$ is analogous to the complex conjugate transpose of the ket. In quantum mechanics the Hilbert space and its basis have a physical interpretation, and this leads directly to perhaps the most counterintuitive aspect of the theory. The counter intuition is this - at the microscopic or quantum level, the state of the system is described by the wave function $\psi$, that is, as a linear superposition of all basis states (i.e. in some sense the system is in all basis states at once). However, at the macroscopic or classical level the system can be in only a single basis state. For example, at the quantum level an electron can be in a superposition of many different energies; however, in the classical realm this obviously cannot be.

\subsection{Coherence and decoherence}

Coherence and decoherence are closely related to the idea of linear superposition. A quantum system is said to be coherent if it is in a linear superposition of its basis states. A result of quantum mechanics is that if a system that is in a linear superposition of states interacts in any way with its environment, the superposition is destroyed. This loss of coherence is called decoherence and is governed by the wave function $\psi$. The coefficients $c_{i}$ are called probability amplitudes, and $\left|c_{i}\right|^{2}$ gives the probability of $|\psi\rangle$ collapsing into state $\left|\phi_{i}\right\rangle$ if it decoheres. Note that the wave function $\psi$ describes a real physical system that must collapse to exactly one basis state. Therefore, the probabilities governed by the amplitudes $c_{i}$ must sum to unity. This necessary constraint is expressed as the unitarity condition

$$
\sum_{i}\left|c_{i}\right|^{2}=1
$$

In the Dirac notation, the probability that a quantum state $|\psi\rangle$ will collapse into an eigenstate $\left|\phi_{i}\right\rangle$ is written $\left.\left\langle\phi_{i} \mid \psi\right\rangle\right|^{2}$ and is analogous to the dot product (projection) of two vectors. Consider, for example, a discrete physical variable called spin. The simplest spin system is a two-state system whose basis states are usually represented as $|\uparrow\rangle$ (spin up) and $|\downarrow\rangle$ (spin down). In this simple system the wave function $\psi$ is a distribution over two values (up and down) and a coherent state $|\psi\rangle$ is a linear superposition of $|\uparrow\rangle$ and $|\downarrow\rangle$. One such state might be

$$
|\psi\rangle=\frac{2}{\sqrt{5}}|\uparrow\rangle+\frac{1}{\sqrt{5}}|\downarrow\rangle
$$

As long as the system maintains its quantum coherence it cannot be said to be either spin up or spin down. It is in some sense both at once. Classically, of course, it must be one or the other, and when this system decoheres the result is, for example, the $|\uparrow\rangle$ state with probability

$$
|\langle\uparrow \mid \psi\rangle|^{2}=\left(\frac{2}{\sqrt{5}}\right)^{2}=0.8
$$

A simple two-state quantum system, such as the one just introduced, is used as the basic unit of quantum computation. Such a system is referred to as a quantum bit or qubit and renaming the two states $|0\rangle$ and $|1\rangle$, it is easy to see why this is so.

\subsection{Operators}

Operators on a Hilbert space describe how one wave function is changed into another. Here they will be denoted by a capital letter with a hat, such as $\hat{A}$, and they may be represented as matrices acting on vectors. Using operators, an eigenvalue equation can be written $\hat{A}\left|\phi_{i}\right\rangle=a_{i}\left|\phi_{i}\right\rangle$, where $a_{i}$ is the eigenvalue. The solutions $\left|\phi_{i}\right\rangle$ to such an equation are called eigenstates and can be used to construct the basis of a Hilbert space as discussed in Section 2.1. In the quantum formalism, all properties are represented as operators whose eigenstates are the basis for the Hilbert space associated with that property and whose eigenvalues are the quantum allowed values for that property. It is important to note that operators in quantum mechanics must be linear operators and further that they must be unitary so that $\hat{A}^{\dagger} \hat{A}=\hat{A} \hat{A}^{\dagger}=\hat{I}$, where $\hat{I}$ is the identity operator and $\hat{A}^{\dagger}$ is the complex conjugate transpose, of $\hat{A}$.

\subsection{Interference}

Interference is a familiar wave phenomenon. Wave peaks that are in phase interfere constructively (magnify each other's amplitude) while those that are out of phase interfere destructively (decrease or eliminate each other's amplitude). This is a phenomenon common to all kinds of wave mechanics from water waves to optics. The well known double slit experiment demonstrates empirically that at the quantum level interference also applies to the probability waves of quantum mechanics. As a simple example, suppose that the wave function described in Section 2.2 is represented in vector form as

$$
|\psi\rangle=\frac{1}{\sqrt{5}}\left(\begin{array}{l}
2 \\
1
\end{array}\right)
$$

and suppose that it is operated upon by an operator $\hat{O}$ described by the following matrix,

$$
\hat{O}=\frac{1}{\sqrt{2}}\left[\begin{array}{cc}
1 & 1 \\
1 & -1
\end{array}\right]
$$

The result is

$$
\hat{O}|\psi\rangle=\frac{1}{\sqrt{2}}\left[\begin{array}{cc}
1 & 1 \\
1 & -1
\end{array}\right] \frac{1}{\sqrt{5}}\left(\begin{array}{l}
2 \\
1
\end{array}\right)=\frac{1}{\sqrt{10}}\left(\begin{array}{l}
3 \\
1
\end{array}\right)
$$


and therefore now

$$
|\psi\rangle=\frac{3}{\sqrt{10}}|\uparrow\rangle+\frac{1}{\sqrt{10}}|\downarrow\rangle
$$

Notice that the amplitude of the $|\uparrow\rangle$ state has increased while the amplitude of the $|\downarrow\rangle$ state has decreased. This is due to the wave function interfering with itself through the action of the operator - the different parts of the wave function interfere constructively or destructively according to their relative phases just like any other kind of wave.

To summarize, quantum computation can be defined as representing the problem to be solved in the language of quantum states and producing operators that drive the system to a final state such that when the system is observed there is a high probability of finding a solution.

\section{Quantum Neural Computing}

Researchers are beginning to investigate the potential for combining quantum computation with (classical) neural computation. An interesting set of mathematical analogies between neural network theory and quantum computation has been presented by Perus [6]. Narayanan and Meneer have simulated classical and various approaches to quantum neural networks, comparing their performances [7]. Their work suggests that there are indeed certain types of problems for which quantum neural networks will prove superior to classical ones. Hogg has extended the work of Grover to demonstrate applications for quantum search and optimization in the context of combinatorial search, something common in computational learning methods [8]. This immediately suggests the possibility of an interesting if modest speedup $[O(\sqrt{N})]$ of existing classical algorithms based on combinatorial search. Other relevant work includes quantum decision making [9], which combines classical and quantum neural networks; alternative quantum learning models [10], which again demonstrate that quantum learning algorithms are theoretically provably superior to classical ones in certain situations; quantum Hopfield networks [11]; and quantum associative memories [12] [13] [14]. Also, preliminary work has been done considering quantum competitive learning [15] and learning of quantum operators [16].

\section{Entanglement}

Entanglement is the potential for quantum systems to exhibit correlations that cannot be accounted for classically. From a computational standpoint, entanglement seems intuitive enough - it is simply the fact that correlations can exist between different qubits - for example if one qubit is in the $|1\rangle$ state, another will be in the $|1\rangle$ state. However, from a physical standpoint, entanglement is little understood. The questions of what exactly it is and how it works are still not resolved. What makes it so powerful (and so little understood) is the fact that since quantum states exist as superpositions, these correlations exist in superposition as well. When the superposition is destroyed, the proper correlation is somehow communicated between the qubits, and it is this "communication" that is the crux of entanglement. Mathematically, entanglement may be described using the density matrix formalism. The density matrix $\rho_{\psi}$ of a quantum state $|\psi\rangle$ is defined as

$$
\rho_{\psi}=|\psi\rangle\langle\psi|
$$

For example, the quantum state

$$
|\xi\rangle=\frac{1}{\sqrt{2}}|00\rangle+\frac{1}{\sqrt{2}}|01\rangle
$$

appears in vector form as

$$
|\xi\rangle=\frac{1}{\sqrt{2}}\left(\begin{array}{l}
1 \\
1 \\
0 \\
0
\end{array}\right)
$$

and it may also be represented as the density matrix

$$
\rho_{\xi}=|\xi\rangle\langle\xi|=\frac{1}{2}\left(\begin{array}{cccc}
1 & 1 & 0 & 0 \\
1 & 1 & 0 & 0 \\
0 & 0 & 0 & 0 \\
0 & 0 & 0 & 0
\end{array}\right)
$$

while the state

$$
|\psi\rangle=\frac{1}{\sqrt{2}}|00\rangle+\frac{1}{\sqrt{2}}|11\rangle
$$

is represented as

$$
\rho_{\psi}=|\psi\rangle\langle\psi|=\frac{1}{2}\left(\begin{array}{cccc}
1 & 0 & 0 & 1 \\
0 & 0 & 0 & 0 \\
0 & 0 & 0 & 0 \\
1 & 0 & 0 & 1
\end{array}\right)
$$

and the state

$$
|\zeta\rangle=\frac{1}{\sqrt{3}}|00\rangle+\frac{1}{\sqrt{3}}|01\rangle+\frac{1}{\sqrt{3}}|11\rangle
$$

is represented as 


$$
\rho_{\zeta}=|\zeta\rangle\langle\zeta|=\frac{1}{3}\left(\begin{array}{llll}
1 & 1 & 0 & 1 \\
1 & 1 & 0 & 1 \\
0 & 0 & 0 & 0 \\
1 & 1 & 0 & 1
\end{array}\right)
$$

where the matrices and vectors are indexed by the state labels $00, \ldots, 11$. Now, notice that $\rho_{\xi}$ can be factorized as

$$
\rho_{\xi}=\frac{1}{2}\left(\left(\begin{array}{ll}
1 & 0 \\
0 & 0
\end{array}\right) \otimes\left(\begin{array}{ll}
1 & 1 \\
1 & 1
\end{array}\right)\right)
$$

where $\otimes$ is the normal tensor product. On the other hand, $\rho_{\psi}$ can not be factorized. States that can not be factorized are said to be entangled, while those that can be factorized are not. Notice that $\rho_{\zeta}$ can be partially factorized two different ways, one of which is

$$
\rho_{\zeta}=\frac{1}{3}\left(\left(\begin{array}{ll}
1 & 1 \\
1 & 1
\end{array}\right) \otimes\left(\begin{array}{ll}
0 & 0 \\
0 & 1
\end{array}\right)+\left(\begin{array}{llll}
1 & 1 & 0 & 1 \\
1 & 0 & 0 & 0 \\
0 & 0 & 0 & 0 \\
1 & 0 & 0 & 0
\end{array}\right)\right)
$$

(the other contains the factorization of $\rho_{\xi}$ and a different remainder); however, in both cases the factorization is not complete. Therefore, $\rho_{\zeta}$ is also entangled, but not to the same degree as $\rho_{\psi}$ (because $\rho_{\zeta}$ can be partially factorized but $\rho_{\psi}$ cannot). Thus there are different degrees of entanglement and much work has been done on better understanding and quantifying it [17] [18]. It is interesting to note from a computational standpoint that quantum states that are superpositions of only basis states that are maximally far apart in terms of Hamming distance are those states with the greatest entanglement. For example, $\rho_{\psi}$ is a superposition of only the states 00 and 11 , which have a maximum Hamming spread, and therefore $\rho_{\psi}$ is maximally entangled. Finally, it should be mentioned that while interference is a quantum property that has a classical cousin, entanglement is a completely quantum phenomenon for which there is no classical analog.

\section{Entanglement in Quantum Neural Computing}

As an example of the power of entanglement, consider a periodic function $f$ with period $r$. Suppose that we have access to a quantum computer with two quantum registers of length $n$ and $m$ respectively, initially in the state $\left|0^{n}\right\rangle\left\langle 0^{m}\right\rangle$. Further suppose that we know a quantum operator $\hat{F}$ for calculating $f$, taking the input from the first register and putting the output in the second register. We can load the first register with a superposition of all possible states, representing all possible inputs (of length $n$ ). This gives

$$
\left(\frac{1}{2^{n}} \sum_{x=0}^{2^{n}-1}|x\rangle\right)\left|0^{m}\right\rangle
$$

The parenthesis are not necessary in the above expression but are used to emphasize that the two registers are not entangled at this point. In other words, knowing a value in one of the registers gives no information about the value in the other. We can then apply $\hat{F}$ to the two registers, effectively computing the value of $f$ for all inputs in parallel

$$
\frac{1}{2^{n}} \sum_{x=0}^{2^{n}-1}(|x\rangle|f(x)\rangle)
$$

Finally, we can observe (only!) the second register causing it to collapse to one of its basis states, in this case to one of the periodic functional values $|k\rangle$. Due to entanglement, the first register will also be affected, even though we do not directly observe it. The resulting quantum state is

$$
\left(\frac{r}{2^{n}} \sum_{y \mid f(y)=k}|y\rangle\right)|k\rangle
$$

revealing the period $r$ (within an additive constant) of $f$. Notice that again the two registers are not entangled. This process is a vital part of several interesting quantum algorithms, most notably Shor's famous algorithm for prime factorization. The key to the process is the entanglement generated in the system by applying the $\hat{F}$ operator, producing correlations between different parts of the system (between the input and output registers in this case).

In neural computing, correlations between parts of the system are typically effected by weighted connections between processing elements. For example, consider the simple feed forward classifier of Figure 1.



Figure 1: Simple feedforward classifier network.

Choosing an input vector $x$ for the network determines the values of the hidden layer units $h$ via the weight matrix $W$, 
which in turn determines the values for the output vector $y$ via the weight matrix $Z$ (thresholding shown in the nodes). We can write this as $y=Z \tau(W x)$ where $\tau$ is a thresholding function. The combination of connection weights and thresholding functions describes the correlations in the network. This network computes the XOR function, and for example, the input $x=11$ results in the output

$$
y=\left[\begin{array}{ll}
-1 & 1
\end{array}\right] \tau\left(\left[\begin{array}{ll}
1 & 1 \\
1 & 1
\end{array}\right]\left(\begin{array}{l}
1 \\
1
\end{array}\right)\right)=\left[\begin{array}{ll}
-1 & 1
\end{array}\right] \tau\left(\left(\begin{array}{l}
2 \\
2
\end{array}\right)\right)=\left[\begin{array}{ll}
-1 & 1
\end{array}\right]\left(\begin{array}{l}
1 \\
1
\end{array}\right)=0
$$

Alternatively, three qubits in the entangled state

$$
|\phi\rangle=\frac{1}{2}|000\rangle+\frac{1}{2}|011\rangle+\frac{1}{2}|101\rangle+\frac{1}{2}|110\rangle
$$

can also be interpreted as computing the XOR function (a quantum algorithm for producing such an entangled state is given in [19]). The first two qubits encode the input and the third encodes the output. The requisite correlations for computing the function are encoded in the entanglement of the state. Computing the value for the input $x$ requires forcing the first two qubits to have a high probability of being found in the basis state $|x\rangle$. This can be done probabilistically (in this case with a $25 \%$ chance of success) by simply measuring the first two qubits, forcing them to collapse to a basis state. If they are measured and found in the state $|x\rangle$, then due to entanglement, the value of the third qubit will be $|\operatorname{XOR}(x)\rangle$ with unit probability. The probability of finding the input qubits in the $|x\rangle$ state can be improved to unity if the operator

$$
\hat{R}_{x}=r_{i j}\left\{\begin{array}{cc}
1 & i=j \text { and } i \neq x z \\
-1 & i=j \text { and } i=x z \\
0 & \text { otherwise }
\end{array}\right.
$$

followed by the operator

$$
\hat{D}=\left[\begin{array}{cccccccc}
-\frac{1}{2} & 0 & 0 & \frac{1}{2} & 0 & \frac{1}{2} & \frac{1}{2} & 0 \\
0 & 0 & 0 & 0 & 0 & 0 & 0 & 0 \\
0 & 0 & 0 & 0 & 0 & 0 & 0 & 0 \\
\frac{1}{2} & 0 & 0 & -\frac{1}{2} & 0 & \frac{1}{2} & \frac{1}{2} & 0 \\
0 & 0 & 0 & 0 & 0 & 0 & 0 & 0 \\
\frac{1}{2} & 0 & 0 & \frac{1}{2} & 0 & -\frac{1}{2} & \frac{1}{2} & 0 \\
\frac{1}{2} & 0 & 0 & \frac{1}{2} & 0 & \frac{1}{2} & -\frac{1}{2} & 0 \\
0 & 0 & 0 & 0 & 0 & 0 & 0 & 0
\end{array}\right]
$$

is applied to the state $|\phi\rangle$ before measuring the input qubits. Here the rows and columns of $\hat{R}_{x}$ and $\hat{D}$ are labeled with binary strings corresponding to the basis states of the quantum system; $i, j, x$, and $z$ are binary strings; and $i=x z$ means that the binary string $i$ is equal to the binary string $x$ concatenated with the binary string $z$. Thus

$$
|y\rangle=\hat{D} \hat{R}_{x}|\phi\rangle
$$

effects the same functionality as the neural network representation above. Repeating the example of computing the output for $x=11$,

$$
\hat{R}_{11}=\left[\begin{array}{cccccccc}
1 & 0 & 0 & 0 & 0 & 0 & 0 & 0 \\
0 & 1 & 0 & 0 & 0 & 0 & 0 & 0 \\
0 & 0 & 1 & 0 & 0 & 0 & 0 & 0 \\
0 & 0 & 0 & 1 & 0 & 0 & 0 & 0 \\
0 & 0 & 0 & 0 & 1 & 0 & 0 & 0 \\
0 & 0 & 0 & 0 & 0 & 1 & 0 & 0 \\
0 & 0 & 0 & 0 & 0 & 0 & -1 & 0 \\
0 & 0 & 0 & 0 & 0 & 0 & 0 & -1
\end{array}\right]
$$

and therefore,

$$
\begin{aligned}
& |y\rangle=\hat{D}\left[\begin{array}{cccccccc}
1 & 0 & 0 & 0 & 0 & 0 & 0 & 0 \\
0 & 1 & 0 & 0 & 0 & 0 & 0 & 0 \\
0 & 0 & 1 & 0 & 0 & 0 & 0 & 0 \\
0 & 0 & 0 & 1 & 0 & 0 & 0 & 0 \\
0 & 0 & 0 & 0 & 1 & 0 & 0 & 0 \\
0 & 0 & 0 & 0 & 0 & 1 & 0 & 0 \\
0 & 0 & 0 & 0 & 0 & 0 & -1 & 0 \\
0 & 0 & 0 & 0 & 0 & 0 & 0 & -1
\end{array}\right] \frac{1}{2}\left(\begin{array}{c}
1 \\
0 \\
0 \\
1 \\
0 \\
1 \\
1 \\
0
\end{array}\right) \\
& \left.=\left[\begin{array}{cccccccc}
-\frac{1}{2} & 0 & 0 & \frac{1}{2} & 0 & \frac{1}{2} & \frac{1}{2} & 0 \\
0 & 0 & 0 & 0 & 0 & 0 & 0 & 0 \\
0 & 0 & 0 & 0 & 0 & 0 & 0 & 0 \\
\frac{1}{2} & 0 & 0 & -\frac{1}{2} & 0 & \frac{1}{2} & \frac{1}{2} & 0 \\
0 & 0 & 0 & 0 & 0 & 0 & 0 & 0 \\
\frac{1}{2} & 0 & 0 & \frac{1}{2} & 0 & -\frac{1}{2} & \frac{1}{2} & 0 \\
\frac{1}{2} & 0 & 0 & \frac{1}{2} & 0 & \frac{1}{2} & -\frac{1}{2} & 0 \\
0 & 0 & 0 & 0 & 0 & 0 & 0 & 0
\end{array}\right] \begin{array}{c}
1 \\
\frac{1}{2} \\
0 \\
0 \\
1 \\
0 \\
1 \\
-1 \\
0
\end{array}\right) \\
& =\left(\begin{array}{l}
0 \\
0 \\
0 \\
0 \\
0 \\
0 \\
1 \\
0
\end{array}\right)=|110\rangle
\end{aligned}
$$


The input qubits are indeed in the basis state $|11\rangle$, and the output qubit is in the appropriate basis state $|0\rangle$. The operators $\hat{R}_{x}$ and $\hat{D}$ are designed to produce the state $|11\rangle$ in the input qubits regardless of the state of the output qubit. Correct computation of the XOR function requires the proper correlation between the input and output qubits. The presence of the appropriate entanglement in the system guarantees this correlation.

In the case of neural networks, changes local to one part of the network (changing a weight or a threshold or an input) can have global effects on the network. Similarly, for entangled quantum states local operations on some qubits indirectly affect the states of all qubits in the system.

\section{Conclusion}

The phenomenon of entanglement in quantum systems can be viewed as playing a role similar to that of weighted connections in a classical neural network, producing correlations between different parts of the system. Entanglement is little understood from a physical standpoint, but computationally it has been identified as playing a key role in providing quantum computation its unique power. The preceding statements, when combined, suggest that quantum computational systems that make use of entangled states have the potential functionality of quantum neural networks. It follows that just as quantum computation is provably superior to classical computation for some problems, it is conceivable that quantum neural networks may prove more powerful than their classical counterparts.

\section{References}

[1] P. Shor, "Polynomial-Time Algorithms for Prime Factorization and Discrete Logarithms on a Quantum Computer", SIAM Journal of Computing, 26(5), 1997, pp. 1484-1509.

[2] L. Grover, "A Fast Quantum Mechanical Algorithm for Database Search", Proceedings of the 28th Annual ACM Symposium on the Theory of Computing, ACM, New York, 1996, pp. 212-219.
[3] D. Simon, "On the Power of Quantum Computation", SIAM Journal of Computing, 26(5), 1997, pp. 1474-1483.

[4] D. Deutsch and R. Jozsa, "Rapid Solution of Problems by Quantum Computation", Proceedings of the Royal Society of London Series A, 439, 1992, pp. 553-558.

[5] R.P. Feynman, R.B. Leighton and M. Sands, The Feynman Lectures on Physics, Addison-Wesley Publishing Company, Massachusetts, 1965.

[6]M. Perus, "Neuro-quantum Parallelism in Brain-mind and Computers", Informatica 20, 1996, pp. 173-183.

[7] A. Narayanan and T. Meneer, "Quantum Artificial Neural Network Architectures and Components", Information Sciences 128, 2000, pp. 231-255.

[8] T. Hogg and D. Portnov, "Quantum Optimization", Information Sciences 128, 2000, pp. 181-197.

[9] M. Zak, "Quantum Decision-maker", Information Sciences 128, 2000, pp. 199-215.

[10] N.H. Bshouty and J. Jackson, "Learning DNF over the Uniform Distribution Using a Quantum Example Oracle", Proceedings of the $8^{\text {th }}$ Annual Conference on Computational Learning Theory, ACM Press, 1995, pp. 118-127.

[11] E.C. Behrman, L.R. Nash, J.E. Steck, V.G. Chandrashekar and S.R. Skinner, "Simulations of Quantum Neural Networks", Information Sciences 128, 2000, pp. 257-269.

[12] D. Ventura and T. Martinez, "Quantum Associative Memory", Information Sciences 124, 2000, pp. 273-296.

[13] A. Ezhov, A. Nifanova and D. Ventura, "Distributed Queries for Quantum Associative Memory", Information Sciences 128, 2000, pp. 271-293.

[14] J. Howell, J. Yeazell and D. Ventura, "Optically Simulating a Quantum Associative Memory", Physical Review A 62, 2000, article \#42303.

[15] D. Ventura, "Implementing Competitive Learning in a Quantum System", Proceedings of the International Joint Conference on Neural Networks, 1999, paper \#513.

[16] D. Ventura, "Learning Quantum Operators", Proceedings of the International Conference on Computational Intelligence and Neuroscience, 2000 , pp. 750-752.

[17] V. Vedral, M.B. Plenio, M.A. Rippin and P.L. Knight, "Quantifying Entanglement", Physical Review Letters, 78(12), 1997, pp. 2275-2279.

[18] R. Jozsa, "Entanglement and Quantum Computation", The Geometric Universe, eds. S. Hugget, L. Mason, K.P. Tod, T. Tsou and N.M.J. Woodhouse, Oxford University Press, 1998, pp. 369379.

[19] D. Ventura and T. Martinez, "Initializing the Amplitude Distribution of a Quantum State", Foundations of Physics Letters, 12(6), 1999, pp. 547-559. 